\section{Subcutaneous interferon $\beta$-1a administration by electronic auto-injector is associated with high adherence in patients with relapsing remitting multiple sclerosis in a real-life study}

\author{
Elina Järvinen,1 Juha Multanen,2 \\ Sari Atula ${ }^{3}$
}

1Merck Finland, Espoo; ${ }^{2}$ Avokuntoutus Aksoni, Helsinki; ${ }^{3}$ Helsinki University Central Hospital, Helsinki, Finland

\begin{abstract}
The objective was to investigate adherence measured by an electronic auto-injector device, and self-reported adherence and treatment convenience in subjects with relapsing remitting multiple sclerosis (RRMS), using an electronic auto-injector Rebismart $^{\circledR}$ to self-inject interferon $\beta$-1a. Thirty one patients with RRMS using the electronic auto-injector Rebismart ${ }^{\circledR}$ for selfinjecting interferon $\beta$-1a subcutaneously three times weekly were included in a reallife clinical multicenter study for 24 weeks in Finland. Mean adherence reported by the device and mean self-assessment of adherence were studied. Reasons for missing injections and treatment convenience were assessed. Association between adherence and gender and age were studied. The mean adherence calculated from the device data was $93.5 \%$. The mean self-assessment of adherence was $96.6 \%$. The most common reason for missing an injection was forgetfulness. Adherence (measured by the device) was not changed over time. In the high adherence group there were more females and young patients $(<30$ years of age). The auto-injector was found to substantially ease the treatment by $90 \%$ of the patients. The electronic auto-injector was associated with high adherence to treatment. The device was found to ease the patient's treatment and it was perceived as easy to use. It is a convenient tool to assess patient's adherence to treatment.
\end{abstract}

\section{Introduction}

Multiple sclerosis (MS) is a chronic neuroinflammatory disease affecting the central nervous system (CNS), and a common cause of disability in young adults. ${ }^{1}$ Relapsing remitting MS (RRMS) is characterized by acute attacks, relapses, which lead to new or increasing neurologic dysfunction and disease worsening. ${ }^{2}$ Therapies used for treatment of RRMS aim to reduce relapse frequency and severity and control disease progression. Disease modifying treatments are administered by subcutaneous (s.c.) or intramuscular injections, orally or by intravenous infusions. ${ }^{3}$

In a chronic disabling disorder such as MS, adherence to treatment is of critical importance in maximizing benefits over the long term. However, it has been shown that non-adherence to treatment in RRMS is common, and poor adherence or treatment gaps are associated with a higher rate of relapses.4,5 For immunomodulatory treatments administered by frequent s.c. injections, injection stress and pain or injection site reactions are reported reasons for nonadherence.6-8 Mechanical auto-injector devices have been shown to improve injection tolerability compared to manual s.c. injections ${ }^{9}$ and the usage of electronic autoinjector device has been shown to lead to high adherence to interferon $\beta$-1a treatment in observational studies. $8,10-12$

Here we were interested in studying the difference between adherence measured by the electronic auto-injector, and self-reported adherence by using the electronic RebiSmart ${ }^{\circledR}$ auto-injector, which automatically records injections, and we addressed self-reported adherence and treatment convenience by a questionnaire in a real-life clinical multicenter setting in Finland.

\section{Materials and Methods}

\section{Study design}

This was an observational, national, multicenter study. Outcome measures studied were: adherence measured by the electronic auto-injector, self-reported adherence by answering a questionnaire and the differences between these, the distribution of different adherence levels, treatment convenience, reasons for missed injections, selfreported adherence over time and association between adherence and gender, age, expanded disability status scale (EDSS).

The study was approved by the local ethics committee. The study included two visits, at baseline and at 24 weeks. At the baseline visit those patients who were using interferon $\beta$-1a s.c. three times weekly (tiw) and had chosen the electronic auto-injector independently of the study entry, and had come to either a patient training visit (new auto-injector users) or to a normal control visit (patients using auto-injector before study entry) were asked for their consent, and the patients signed a written consent.
Correspondence: Sari Atula, Helsinki University Central Hospital, Haartmaninkatu 4, 00290 Helsinki, Finland.

Tel: +358.40 .8339932 .

E-mail: sari.atula@hus.fi

Key words: Relapsing remitting multiple sclerosis; Adherence; Auto-injector; Interferon $\beta$ - $1 \mathrm{a}$.

Contributions: EJ: interpretation of the data, drafting of the manuscript; JM: design and coordination of the study, drafting of the manuscript; SA: design of the study, collection of the material, analysis and interpretation of the data, drafting of the manuscript.

Conflict of interest: EJ is an employee of Merck Finland, an affiliate of Merck KGaA Darmstadt, Germany. SA declares no conflict of interest. JM declares no conflict of interest.

Funding: funding for this work was provided by Merck Finland, a business of Merck KGaA, Darmstadt, Germany.

Acknowledgements: we thank Jorma Sormunen for the design of the study and Marketta Kause (Merck Finland) for the study coordination. We thank Liisa Luostarinen, MD, Sari Rastas, MD, Irma Keskinarkaus, MD, Keijo Koivisto, MD, Ibrahim Mahjneh, MD, and Elena Haapaniemi, MD for recruiting patients to the study and Sari Järvisalo, RN for conducting the study.

Received for publication: 7 November 2016 Accepted for publication: 15 January 2017.

This work is licensed under a Creative Commons Attribution NonCommercial 4.0 License (CC BY-NC 4.0).

CCopyright E. Järvinen et al., 2017

Licensee PAGEPress, Italy

Neurology International 2017; 9:6957

doi:10.4081/ni.2017.6957

All eligible patients consented to participate in the study.

At the 24-week visit, the adherence to the treatment was assessed by the investigator or MS nurse by reading the device calendar. Adherence was assessed at different time periods (baseline-week 4, weeks 5-8, weeks 9-12, weeks 13-16, weeks 17-20 and weeks 21-24) by the questionnaire. Also, patients filled in a convenience questionnaire evaluating their own experience of using the device. Potential adverse events and product defects were reported according to the standard guidelines.

\section{Patients}

The patients were eligible for the study if they were diagnosed with RRMS according to the McDonald criteria (2005), ${ }^{10}$ naïve 
to treatment or currently being treated with interferon $\beta$-1a s.c. tiw injected by RebiSmart $^{\circledR}$ auto-injector for no longer than nine months prior to enrollment, meeting the inclusion criteria, and independently scheduled to receive interferon $\beta$-1a $22 / 44 \mathrm{~g}$ s.c. tiw via the auto-injector. Inclusion criteria: males and females, between 18 to 65 years of age, stable disease defined as patient being relapse-free for at least 30 days before the enrollment and $\operatorname{EDSS}<6$.

\section{Statistical analyses}

The study was descriptive. Adherence of the study population was displayed by summary statistics. Adherence (\%) was calculated for each patient as $100 \mathrm{x}$ the number of injections the patient administered divided by the expected number of injections over six months. We studied by descriptive methods whether there is an association between adherence and gender, age and EDSS. Results of the convenience questionnaire were displayed descriptively for each item with the help of a sum score over all items. Reasons for missed injections were collected.

\section{Results}

This was an observational real-life study to assess adherence and convenience of interferon $\beta$-1a s.c. tiw treatment in RRMS patients using an electronic autoinjector. A total of 31 patients with RRMS from eight centers met the inclusion criteria for the study. For two of the patients no administration data could be retrieved, and hence 29 patients were included in the analyses. Patient demographics are shown in Table 1.

\section{Adherence measured by the elec- tronic auto-injector}

The mean adherence calculated from the device data was $93.5 \%(88.4 ; 98.2 ; 95 \%$ CI). A total of $62 \%$ of the patients had a adherence of more than $95 \%$, while only $7 \%$ had adherence of $75 \%$ or less (Figure 1). To assess the possible factors affecting adherence, the demographics of the high adherence (95-100\%) group was investigated. In this high-adherence group there were more female $(72 \%)$ than male patients (55\%). The age groups $30-40$ and $>40$ years had very similar adherence (62 and $64 \%$, respectively), but for young patients (under 30 years) it was slightly higher (75\%). No difference in adherence was seen for patients with a low EDSS score (0-1) compared with those with a higher score (1.5-4); it was $95-100 \%$ for $64 \%$ in both groups.

\section{Self-reported adherence}

The mean adherence calculated from the self-assessed questionnaire was $96.6 \%$ (94.3; 99.0; 95\% CI). The adherence of more than $95 \%$ was reported for $38 \%$ of the patients, while only $3 \%$ had an adherence of $75 \%$ or less (Figure 2). The most reported reason for missing an injection was forgetting, reported by $64 \%$ of the patients providing a reason. The demographics for the high-adherence (95-100\%) group were similar as was seen in the high compliance group measured from the device data. To receive information on whether self-reported adherence was changed during the study, it was assessed during different time periods (Figure 3). There were no changes over time in the proportion of patients with a adherence of more than $75 \%$ (missing $0-3$ injections during 4 weeks).

\section{Factors affecting adherence}

A total of $90 \%$ or more of the patients found it easy to learn to use the auto-injector, to grab and hold the devise, to prepare the devise and to set the drug cartridge. $65 \%$ or more of the patients found it easy to attach and detach the needle, to change the battery, and to keep and carry the devise.

\section{Opinions on the device (RebiSmart ${ }^{\circledR}$ )}

A total of $90 \%$ of the patients found the auto-injector to substantially ease their treatment. The device settings were changed by $52 \%$ of the patients, and $21 \%$ reported that they had to alter the device settings between injections very frequently or often. The most common reasons for this was injection site discomfort and that the dose needed to be adjusted. On the other hand, $88 \%$ found it easy to change the settings. Over $85 \%$ of the patients perceived injecting easy and the preparation time short. More than $65 \%$ of the patients found the devise to be easy to use when travelling, and the signal lights and sounds to ease the treatment.

\section{Discussion}

Here we assessed adherence measured by an electronic auto-injector and selfreported adherence and treatment convenience in RRMS patients using electronic auto-injector RebiSmart ${ }^{\circledR}$. The mean adherence to interferon $\beta$-1a s.c. tiw, as calculated from the auto-injector device, was high $(94 \%)$, and in line with other studies where interferon $\beta$-1a s.c. tiw has been injected by an auto-injector.8,10-14 The overall patients' self-estimation of adherence (mean) was
Table 1. Patients' demographics and baseline characteristics.

\begin{tabular}{lc}
\hline Age (years) & \\
N 29 & $39(8.0)$ \\
Mean (SD) & $39(23-50)$ \\
Median (range) & \\
Gender, N (\%) & $18(62)$ \\
$\quad$ Female & $11(38)$ \\
Male & \\
Ethnic origin & $29(100)$ \\
$\quad$ Caucasian & \\
EDSS score, N (\%) & $11(38)$ \\
$0-1$ & $12(41)$ \\
1.5-4 & 6 \\
Missing &
\end{tabular}

Earlier experience with RebiSmart® ( $<9$ months)
Yes
$6(21)$

No

$23(79)$

$\mathrm{N}$, number; SD, standard deviation; EDSS, expanded disability status scale.

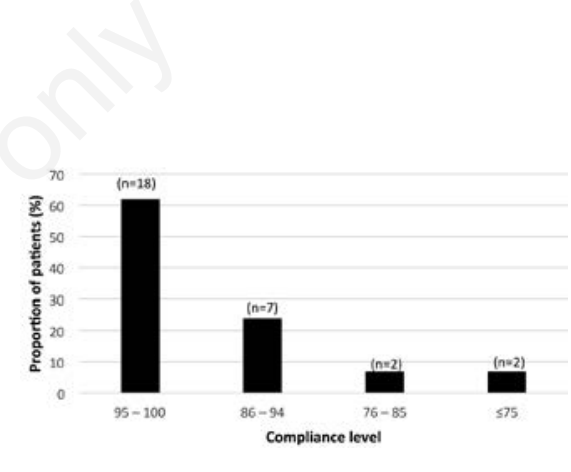

Figure 1. Adherence to treatment calculated from device data.

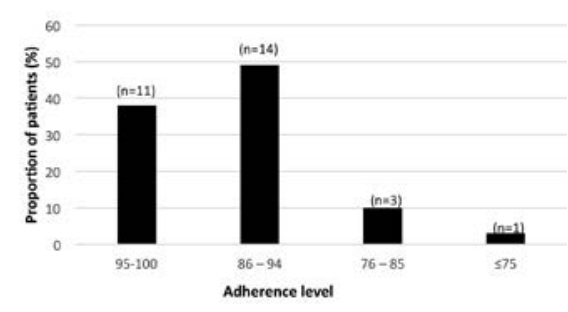

Figure 2. Self-assessment of adherence to treatment by answering a convenience questionnaire.

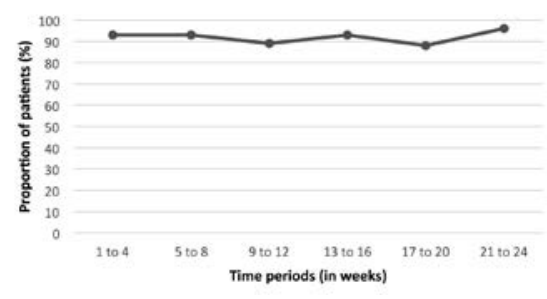

Figure 3. Proportion of patients with specified self-reported adherence within 4 weeks. 
$96.6 \%$ and thus higher than the values recorded by the device. Although the difference was small, likely because of the small number of subjects in the study, this implicates that the difference may cause unnecessary switches between treatments, as nonadherence leads to lack of efficacy. The electronic auto-injector is a convenient tool to assess patient's adherence to treatment, and its use may thus have important implications in determining patient care.

Demographic factors associated with adherence to treatment were sex and age of the patient. In the high adherence group there were more females than males, and more young patients. Young people may generally adapt new technologies more easily. In some studies males have been more adherent, ${ }^{15,16}$ but also studies with no differences has been reported. 8,17 We did not observe any difference in adherence between patients with low and high EDSS. By using the electronic auto-injector injection site reactions and tolerability can be improved.9,17-19 In our study only one patient stopped the treatment due to an adverse event, pointing at a good tolerability. In addition, a vast majority ( $90 \%$ ) of the patients answered that the device substantially eases their treatment, which can be an explanation to the high adherence. Injecting was perceived easy and the preparation time for the injection short. The cartridge setting, and attaching and detaching of the needle was easy to a majority of the patients.

Adherence may decrease over time, which was not observed here. However, the study period was relatively short, and we cannot exclude the possibility that during a longer observation period a change could be detected. Limitations of this study include the small number of subjects, short followup and the open-label nature of the study. However, real-life data including objective information on adherence, and data on the differences between adherence measured by the electronic auto-injector and self-reported adherence could be obtained.

\section{Conclusions}

In conclusion, the electronic RebiSmart $^{\circledR}$ auto-injector for the self-injection of interferon $\beta$-1a s.c. tiw was associated with high adherence to treatment. The device was found to ease the patient's treatment and it was perceived as easy to use by the patients. Moreover, electronic autoinjector is a convenient tool to assess patient's true adherence.

\section{References}

1. Dendrou CA, Fugger L, Friese MA. Immunopathology of multiple sclerosis. Nat Rev Immunol 2015;15:545-58.

2. Lublin FD. New multiple sclerosis phenotypic classification. Eur Neurol 2014;72(Suppl.1):1-5.

3. Wiendl H, Meuth SG. Pharmacological approaches to delaying disability progression in patients with multiple sclerosis. Drugs 2015;75:947-77.

4. Boyko AN. Clinical effects and tolerability of high-dose, high-frequency recombinant interferon beta- $1 \mathrm{a}$ in patients with multiple sclerosis: maximizing therapy through long-term adherence. Expert Opin Biol Ther 2010;10:653-66.

5. Steinberg SC, Faris RJ, Chang CF, et al. Impact of adherence to interferons in the treatment of multiple sclerosis: a non-experimental, retrospective, cohort study. Clin Drug Investig 2010;30:89100.

6. Brandes DW, Callender T, Lathi E, O'Leary S. A review of disease-modifying therapies for MS: maximizing adherence and minimizing adverse events. Curr Med Res Opin 2009;25:7792.

7. Devonshire VA, Verdun di Cantogno E. Review of subcutaneous interferon $\beta$ 1a, delivered via the electronic selfinjection device RebiSmart ${ }^{\mathrm{TM}}$, for the treatment of multiple sclerosis. Ther Deliv 2011;2:1455-65.

8. Bayas A, Ouallet JC, Kallmann B, et al. SMART study group. Adherence to, and effectiveness of, subcutaneous interferon $\beta$-1a administered by RebiSmart $\AA$ in patients with relapsing multiple sclerosis: results of the 1-year, observational SMART study. Expert Opin Drug Deliv 2015;12:1239-50.

9. Mikol D, Lopez-Bresnahan M, Taraskiewicz S, et al. A randomized, multicentre, open-label, parallel-group trial of the tolerability of interferon beta-1a (Rebif) administered by autoinjection or manual injection in relapsingremitting multiple sclerosis. Mult Scler 2005;11:585-91.

10. Fernández O, Arroyo R, MartínezYélamos $\mathrm{S}$, et al. long-term adherence to ifn beta-1a treatment when using RebiSmart ${ }^{\circledR}$ device in patients with relapsing-remitting multiple sclerosis. PLoS One 2016;11:e0160313.

11. Devonshire VA, Feinstein A, Moriarty P. Adherence to interferon $\beta$-1a therapy using an electronic self-injector in multiple sclerosis: a multicentre, singlearm, observational, phase IV study. BMC Res Notes 2016;9:148.

12. Lugaresi A, De Robertis F, Clerico M, et al. Long-term adherence of patients with relapsing-remitting multiple sclerosis to subcutaneous self-injections of interferon $\beta$-1a using an electronic device: the RIVER study. Expert Opin Drug Deliv 2016;13:931-5.

13. Polman CH, Reingold SC, Edan G, et al. Diagnostic criteria for multiple sclerosis: 2005 revisions to the "McDonald Criteria”. Ann Neurol 2005;58:840-6.

14. Willis H, Webster J, Larkin AM, Parkes L. An observational, retrospective, UK and Ireland audit of patient adherence to subcutaneous interferon beta-1a injections using the RebiSmart( $(\circledR)$ injection device. Patient Prefer Adherence 2014; 8:843-51.

15. Jongen PJ, Sanders E, Zwanikken C, et al. Adherence to monthly online selfassessments for short-term monitoring: a 1-year study in relapsing-remitting multiple sclerosis patients after start of disease modifying treatment. Patient Prefer Adher 2013;7:293-300.

16. Ivanova JI, Bergman RE, Birnbaum $\mathrm{HG}$, et al. Impact of medication adherence to disease-modifying drugs on severe relapse, and direct and indirect costs among employees with multiple sclerosis in the US. J Med Econ 2012;15:601-9.

17. Singer B, Wray S, Miller $\mathrm{T}$, et al. Patient-rated ease of use and functional reliability of an electronic autoinjector for self-injection of subcutaneous interferon beta-1a for relapsing multiple sclerosis. Mult Scler Relat Disord 2012;1:87-94.

18. Lugaresi A, Florio C, Brescia-Morra V, et al. Patient adherence to and tolerability of self-administered interferon $\beta$-1a using an electronic autoinjection device: a multicentre, open-label, phase IV study. BMC Neurol 2012;12:7.

19. Devonshire V, Arbizu T, Borre B, Lugaresi A, et al. Patient-rated suitability of a novel electronic device for selfinjection of subcutaneous interferon beta-1a in relapsing multiple sclerosis: an international, single-arm, multicentre, Phase IIIb study. BMC Neurol 2010;10:28. 